\title{
Therapeutic novelties in migraine: new drugs, new hope?
}

\author{
Thien Phu Do, Song Guo and Messoud Ashina*
}

\begin{abstract}
Background: In the past decade, migraine research has identified novel drug targets. In this review, we discuss recent data on emerging anti-migraine therapies.

Main body: The development of ditans, gepants and anti-calcitonin gene-related peptide monoclonal antibodies for the treatment of migraine is one of the greatest advances in the migraine field. Lasmiditan, rimegepant and ubrogepant will extend our therapeutic armamentarium for managing acute migraine attacks when triptans are not effective or contraindicated due to cardiovascular disorders. The monoclonal antibodies are migraine specific prophylactic drugs with high responder rates and favorable adverse event profiles. Furthermore, they offer convenient treatment regimens of 4 - or 12-week intervals.
\end{abstract}

Conclusion: Collectively, novel migraine therapies represent a major progress in migraine treatment and will undoubtedly transform headache medicine.

Keywords: Migraine, Randomized clinical trial, Efficacy, Tolerability, Adverse event, Ditan, Gepant, Antibody

\section{Introduction}

The last ten years have witnessed remarkable discoveries in migraine research $[1,2]$ and migraine therapy is currently undergoing tremendous development. Based on these discoveries, novel targeted acute and preventive therapies have emerged including ditans $\left(5-\mathrm{HT}_{1 \mathrm{~F}}\right.$ receptor agonists), gepants (calcitonin gene-related peptide (CGRP) receptor antagonists) and anti-CGRP monoclonal antibodies (mAbs). Novel therapies signify a paradigm shift in the migraine management and bring new hope to patients and clinicians. This review provides an overview of new drugs for both acute and prophylactic treatments of migraine, covering studies on clinical evidence, tolerability, and the different stages of clinical development.

\section{Novel acute treatment targets}

\section{5-HT $T_{1 F}$ receptor agonists (ditans)}

Triptans are $5-\mathrm{HT}_{1 \mathrm{~B}} / 1 \mathrm{D}$ receptor agonists with some affinity for the $5-\mathrm{HT}_{1 \mathrm{~F}}$ receptor subtype and commonly used as acute anti-migraine drugs [3]. The rationale for the development of triptans was based on the vasoconstricting

\footnotetext{
* Correspondence: ashina@dadlnet.dk

Danish Headache Center and Department of Neurology, Rigshospitalet Glostrup, Faculty of Health Sciences, University of Copenhagen, Copenhagen, Denmark
}

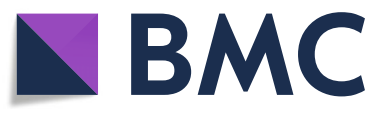

(c) The Author(s). 2019 Open Access This article is distributed under the terms of the Creative Commons Attribution 4.0 International License (http://creativecommons.org/licenses/by/4.0/), which permits unrestricted use, distribution, and reproduction in any medium, provided you give appropriate credit to the original author(s) and the source, provide a link to the Creative Commons license, and indicate if changes were made. effect via the $5-\mathrm{HT}_{1 \mathrm{~B}}$ receptor subtype [4]. However, some studies have questioned the role of vasoconstriction in anti-migraine effect of triptans [5]. Therefore, drug pharmacological studies have focused on the $5-\mathrm{HT}_{1 \mathrm{D}}[6$, 7] and $5-\mathrm{HT}_{1 \mathrm{~F}}$ receptors that do not a vasoconstrictive effect $[8-10]$. These receptors are interesting drug targets as triptans are contraindicated in migraine patients with coexisting cardiovascular disorders [11-15]. The 5- $\mathrm{HT}_{1 \mathrm{D}}$ subtype is expressed in the human trigeminal ganglion and co-localize with CGRP [6]. A phase II trial investigated the efficacy of 5- $\mathrm{HT}_{1 \mathrm{D}}$ agonists, but did not meet its primary endpoints and its development was discontinued [7]. Therefore, drug discovery programs shifted focus to the $5-\mathrm{HT}_{1 \mathrm{~F}}$ subtype. This receptor subtype is located in the trigeminal ganglion, the trigeminal nucleus caudalis and cephalic blood vessels, but importantly, activation of this receptor do not constrict blood vessels [8-10]. Interestingly, sumatriptan and naratriptan binds to the $5-\mathrm{HT}_{1 \mathrm{~F}}$ receptor with a high affinity [9]. Based on these studies, $5-\mathrm{HT}_{1 \mathrm{~F}}$ agonists have been developed and categorized as a new drug class: ditans. Studies of ditans in preclinical models suggested an involvement in the modulation of dural neurogenic inflammation and the trigeminovascular system, establishing the $5-\mathrm{HT}_{1 \mathrm{~F}}$ receptor as a potential target for migraine treatment [16]. Three compounds exist, LY 344864, LY334370 and 
lasmiditan, but only the last two have been tested in humans. While LY334370 demonstrated a clinical effect in a proof of concept study [17], the development of LY334370 was terminated due to hepatic toxicity in animal models [18]. Accordingly, only lasmiditan is still undergoing clinical trials (Table 1).

Lasmiditan is a $5-\mathrm{HT}_{1 \mathrm{~F}}$ receptor agonist [19] which is administrated orally in $50-200 \mathrm{mg}$ doses and it has a $t_{\max }$ of $1.5-2.5 \mathrm{~h}$ [20]. Of the three phase III clinical trials (SAMURAI, NCT02439320 [21]; SPARTAN, NCT02605174 [22]; GLADIATOR, NCT02565186 [23]), to date only one (SAMURAI [24]) has been published [24]. Preliminary data from press releases for the remaining two are presented as following [25, 26] (Fig. 1).

In SAMURAI, 2231 patients were randomized to oral intake of lasmiditan $100 \mathrm{mg}, 200 \mathrm{mg}$ or placebo [24]. This trial excluded patients with known coronary artery disease, clinically significant arrhythmia or uncontrolled hypertension. The percentage of patients with 2-h pain freedom was $28.2 \%$ (vs. placebo, $p<0.001$ ) in the $100 \mathrm{mg}$ group, $32.2 \%$ (vs. placebo, $p<0.001$ ) in the $200 \mathrm{mg}$ group and $15.3 \%$ in the placebo group [27]. The percentage of patients with freedom from most bothersome symptom at 2 -h was $40.9 \%$ (vs. placebo, $p<0.001$ ) in the $100 \mathrm{mg}$ group, $40.7 \%$ (vs. placebo, $p<0.001$ ) in the $200 \mathrm{mg}$ group compared to $29.5 \%$ in the placebo group. The most common adverse events were dizziness and paresthesia and both mild to moderate intensity. Dizziness occurred in $11.9 \%$ of the $100 \mathrm{mg}$ group and $15.4 \%$ of the $200 \mathrm{mg}$ group. Paresthesia occurred in $5.7 \%$ of the $100 \mathrm{mg}$ group and $7.6 \%$ of the $200 \mathrm{mg}$ group compared to $3.1 \%$ and $2.1 \%$ in the placebo group. No serious adverse events occurred.

In SPARTAN, patients were randomized to $50 \mathrm{mg}$, $100 \mathrm{mg}, 200 \mathrm{mg}$ or placebo (number of patients included not reported) [25]. This trial did not exclude patients with known coronary artery disease, clinically significant arrhythmia or uncontrolled hypertension. The percentage of patients with 2-h pain freedom was $28.6 \%$ (vs. placebo, $p=0.003$ ) in the $50 \mathrm{mg}$ group, $31.4 \%$ (vs. placebo, $p<0.001$ ) in the $100 \mathrm{mg}$ group, $38.8 \%$ (vs. placebo, $p<0.001$ ) in the $200 \mathrm{mg}$ group and $21.3 \%$ in placebo group. Percentage of patients with freedom from most bothersome symptom at 2-h was $40.8 \%$ (vs. placebo, $p=0.009$ ) in the $50 \mathrm{mg}$ group, $44.2 \%$ (vs. placebo, $p<0.001$ ) in the $100 \mathrm{mg}$ group, $48.7 \%$ (vs. placebo, $p<0.001$ ) in the $200 \mathrm{mg}$ group and $33.5 \%$ in the placebo group. Adverse events included dizziness, paresthesia, somnolence, fatigue, nausea and lethargy.

Table 1 Overview of ditans in alphabetical order

\begin{tabular}{ll}
\hline Drug & Status \\
\hline Alniditan & Development terminated \\
Lasmiditan (COL-144) & Phase III clinical trials \\
LY-334370 & Development terminated \\
\hline
\end{tabular}

The open-label trial GLADIATOR included patients from the prior SAMURAI and SPARTAN trials [26]. Patients were randomized to receive $100 \mathrm{mg}$ or $200 \mathrm{mg}$ to treat up to eight attacks per month (number of patients included not reported, expected a total of 2580). The primary goal was to evaluate the proportion of patients and attacks associated with any adverse events and specific adverse events. Adverse events occurred in 19\% in the $100 \mathrm{mg}$ group and $20 \%$ in the $200 \mathrm{mg}$ group. The most common adverse events included dizziness and paresthesia.

Collectively, randomized controlled trials (RCTs) support the use of lasmiditan for the acute treatment of migraine. The percentage of patients with 2 -h pain freedom in trials ranges from $28.2-38.8 \%$ (Fig. 1). Furthermore, the therapeutic gain (the placebo-subtracted response) for lasmiditan $200 \mathrm{mg}$ is $16.9-17.5 \%$ which seems similar to sumatriptan of $16-21 \%$ for doses 50 $100 \mathrm{mg}$ (Fig. 2) [28]. Approximately $20 \%$ of patients report adverse events most commonly dizziness and paresthesia after intake of lasmiditan 100-200 mg [26]. Overall, the results of these trials demonstrate that lasmiditan is efficacious and well tolerated in patients with a high level of cardiovascular risk factors. In the future, lasmiditan will likely be approved as second-line treatment if patients failed with triptans or first line anti-migraine treatment in patients with cardiovascular risk (or documented cardiovascular disease). Lasmiditan is expected to be approved by the U.S. Food and Drugs Administration (FDA) in 2019.

\section{CGRP receptor antagonists (gepants)}

Small molecule CGRP receptor antagonists are a novel drug class called gepants (Table 2, Fig. 3). In 2004, the first proof of concept study reported that olcegepant had a clinical effect in humans, but this compound was never commercialized as it cannot be orally administrated [29]. Seven gepants have been developed for the treatment of migraine, but some of the drug development programs have since been terminated [30]. While telcagepant demonstrated a clinical effect, development ceased due to a hepatotoxicity risk [30]. This is believed to be due to a liver toxic metabolite that is not formed by other gepants [31]. Currently, two gepants are in phase III clinical trials for the acute treatment of migraine: rimegepant and ubrogepant (Table 2).

Rimegepant is a CGRP receptor antagonist [32] that is administrated orally with a $75 \mathrm{mg}$ dose and it has a $t_{\max }$ of two hours [33]. Preliminary data from two phase III clinical trials have been reported in press releases but not yet published in peer-reviewed journals [34, 35]. Rimegepant was administrated as a $75 \mathrm{mg}$ oral dose in the two trials (Fig. 4). Preliminary results show that $19.2 \%$ (vs. placebo, $p<0.003$ ) of 543 patients and 19.6\% (vs. placebo, $p<0.001$ ) of 537 patients achieve 2-h pain 


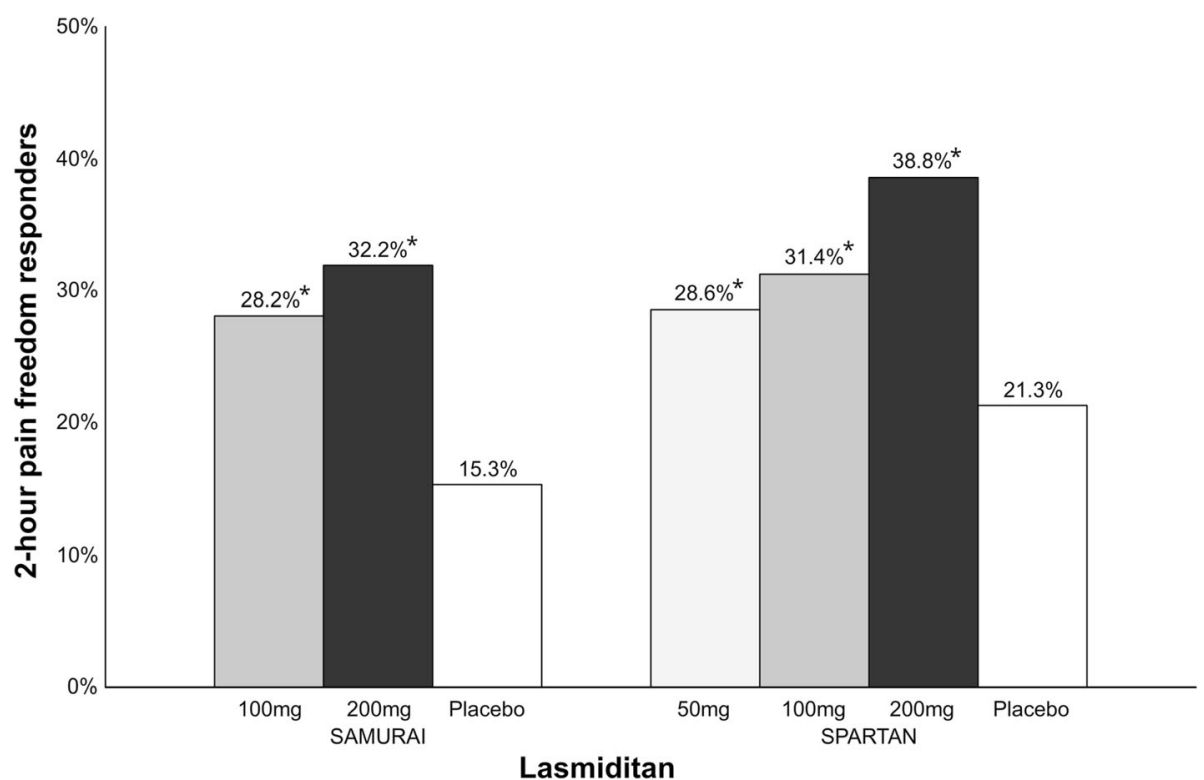

Fig. 1 Overview of patients (\%) achieving 2-h pain freedom in lasmiditan phase III clinical trials with different doses. A darker bar indicates a higher dose. ${ }^{*}$ vs. placebo, $p<0.001$

freedom compared to $14.2 \%$ of 541 patients and $12 \%$ of 535 patients with placebo, respectively [34]. Interestingly, the percentage of patients achieving pain freedom is reported to increase over time with $66 \%$ achieving pain freedom at $8 \mathrm{~h}$ compared to $47 \%$ in placebo group [35]. Freedom from most bothersome symptom was $36.6 \%$ (vs. placebo, $p<0.002$ ) and
$37.6 \%$ (vs. placebo, $p<0.0001$ ) in the two trials compared to $27.7 \%$ and $25.2 \%$ with placebo, respectively. Rimegepant had no effect on hepatic function. The number of patients with adverse events has not been reported from either trial but the most common adverse events were nausea $(1.4 \%$ in active vs. $1.1 \%$ in placebo group) and urinary tract infections

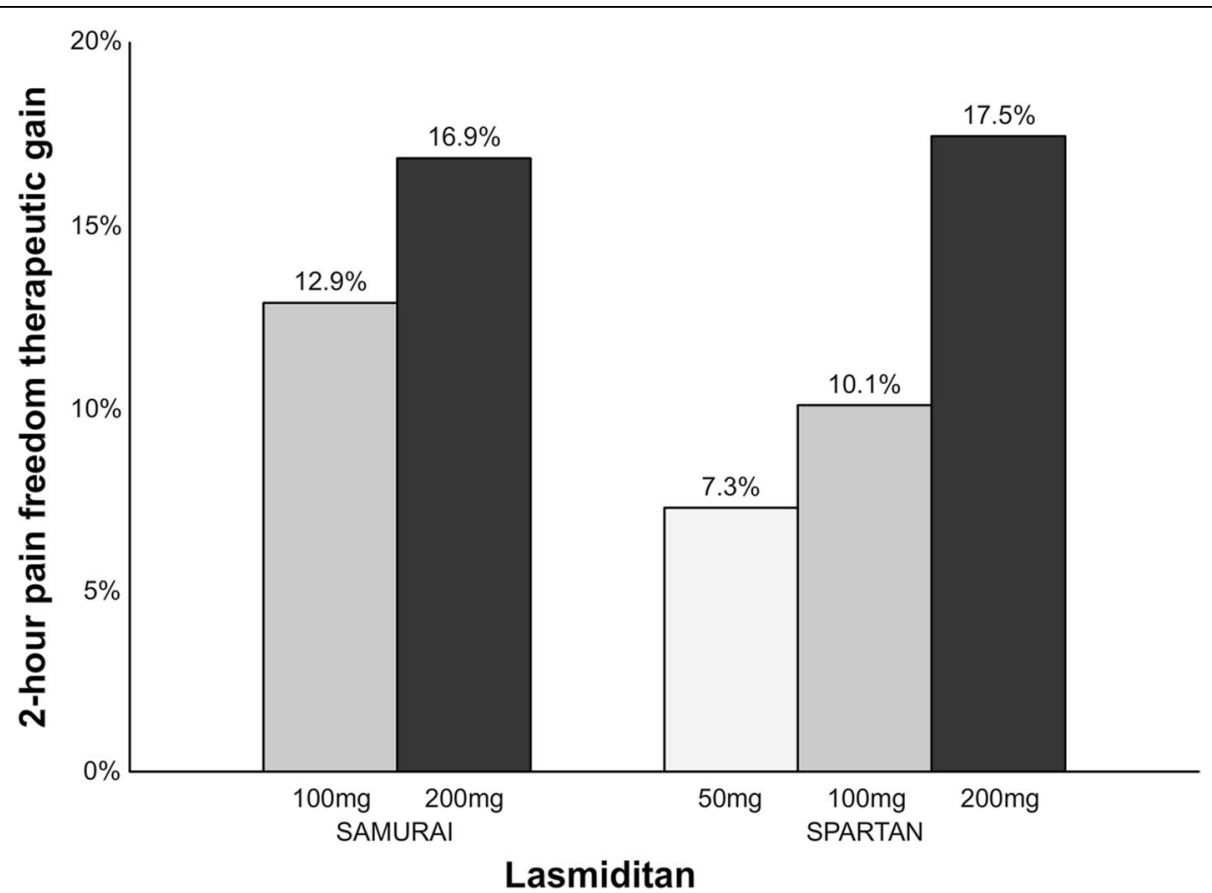

Fig. 2 Overview of the therapeutic gain* in 2-h pain freedom with lasmiditan. A darker bar indicates a higher dose. *Therapeutic gain is defined as the difference between percentage of responders in active group compared to percentage of responders in placebo group 
Table $\mathbf{2}$ Overview of gepants for the treatment of migraine in alphabetical order

\begin{tabular}{ll}
\hline Drug & Status \\
\hline Atogepant (AGN-241689, MK-8031) & Phase III clinical trials (prophylactic treatment) \\
BI 44370 & Development terminated \\
MK-3207 & Development terminated \\
Olcegepant (BIBN4096BS) & Development terminated \\
Rimegepant (BMS-927711, BHV3000) & Phase III clinical trials (acute treatment); phase II clinical trials (prophylactic treatment) \\
Telcagepant (MK-0974) & Development terminated \\
Ubrogepant (MK-1602) & Phase III clinical trials (acute treatment) \\
\hline
\end{tabular}

( $1 \%$ in active vs. $0.7 \%$ in placebo group). Overall adverse event rate is reported to be similar to placebo.

Ubrogepant is a CGRP receptor antagonist that is administrated orally with $25-100 \mathrm{mg}$ doses and it has a $t_{\max }$ of $0.7-1.5 \mathrm{~h}$ [36]. Preliminary data from two phase III clinical trials (ACHIEVE I and ACHIEVE II) have been reported in press releases but not yet published in peer-reviewed journals [37, 38]. In ACHIEVE I, 1327 patients were randomized 1:1:1 to ubrogepant $50 \mathrm{mg}$, ubrogepant $100 \mathrm{mg}$ or placebo (Fig. 5) [37]. The percentage of patients with 2-h pain freedom was $19.2 \%$ (vs. placebo, $p=0.0023$ ) in the $50 \mathrm{mg}$ group, $21.2 \%$ (vs. placebo, $p=0.0003$ ) in the $100 \mathrm{mg}$ group and $11.8 \%$ in the placebo group. Freedom from most bothersome symptom at 2 -h was $38.6 \%$ (vs. placebo, $\mathrm{p}=0.0023$ ) and $37.7 \%$ (vs. placebo, $\mathrm{p}=0.0023$ ) compared to $27.8 \%$ for placebo, respectively. No hepatoxicity was reported after intake of ubrogepant. The most common adverse events were nausea, somnolence, and dry mouth all reported with a frequency lower than 5\%. In ACHIEVE II, 1686 patients were randomized 1:1:1 to ubrogepant $25 \mathrm{mg}$, ubrogepant $50 \mathrm{mg}$ or placebo (Fig. 4) [38]. The percentage of patients with 2-h pain freedom was $20.7 \%$ (vs. placebo, $p=0.0285$ ) in the $25 \mathrm{mg}$ group, $21.8 \%$ (vs. placebo, $p=0.0129$ ) in the $50 \mathrm{mg}$ group and $14.3 \%$ in the placebo group. Freedom from most bothersome symptom at 2 -h was $34.1 \%$ (vs. placebo, $p=0.0711$ ) and $38.9 \%$ (vs. placebo, $\mathrm{p}=0.0129$ ), respectively, compared to $27.4 \%$ for placebo with the 25 mg dose not being statistically significant compared to placebo. There was no signal of hepatic toxicity in this trial. The most common adverse events were nausea and dizziness all reported with a frequency lower than $2.5 \%$.

Collectively, RCTs demonstrated efficacy of gepants for the acute treatment of migraine. The percentage of patients with 2-h pain freedom ranges from 19.2-19.6\% with rimegepant and 19.2-21.8\% with ubrogepant. However, the therapeutic gain for gepants (rimegepant: 5\%-7.6\%; ubrogepant: 6.4\%-9.4\%) (Fig. 6) seems to be low, especially compared to sumatriptan $(16 \%-21 \%$ [28]) and lasmiditan (7.3\%-17.5\%) (Fig. 2). In addition, it is lower compared to the therapeutic gain of telcagepant (17\% in doses $280-300 \mathrm{mg}$ ) [39] and it is unlikely due unoptimized dosage or absorption rate [40]. Previous trials of gepants caused concerns regarding the hepatic safety, but single treatments with rimegepant and ubrogepant were not associated with hepatotoxicity. Since gepants do not constrict cranial arteries [4143], they, like ditans, can be used as first line antimigraine treatment in patients with cardiovascular
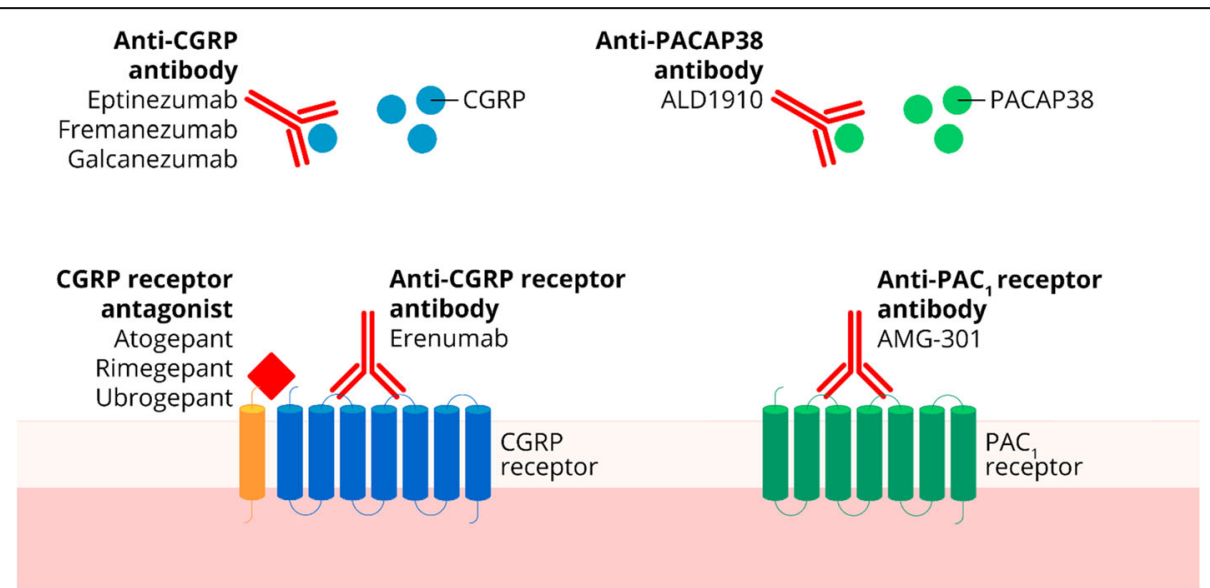

Fig. 3 Overview of the therapeutic novelties targeting the calcitonin gene-related peptide (CGRP) and pituitary adenylate cyclase-activating polypeptide/pituitary adenylate cyclase 1 (PACAP/PAC $)$ pathways developed for migraine 


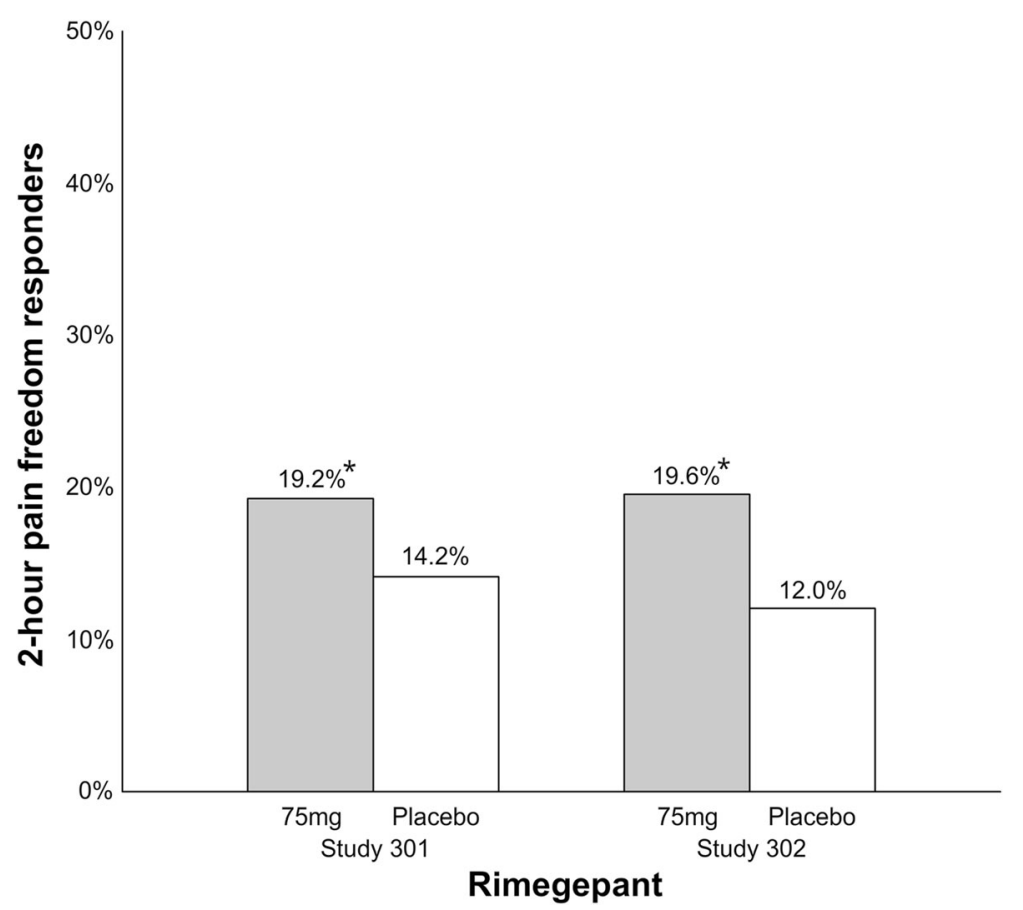

Fig. 4 Overview of patients (\%) achieving 2-h pain freedom in rimegepant phase III clinical trials. *Study 301; vs. placebo, $p<0.003$. Study 302 ; vs. placebo, $p<0.001$

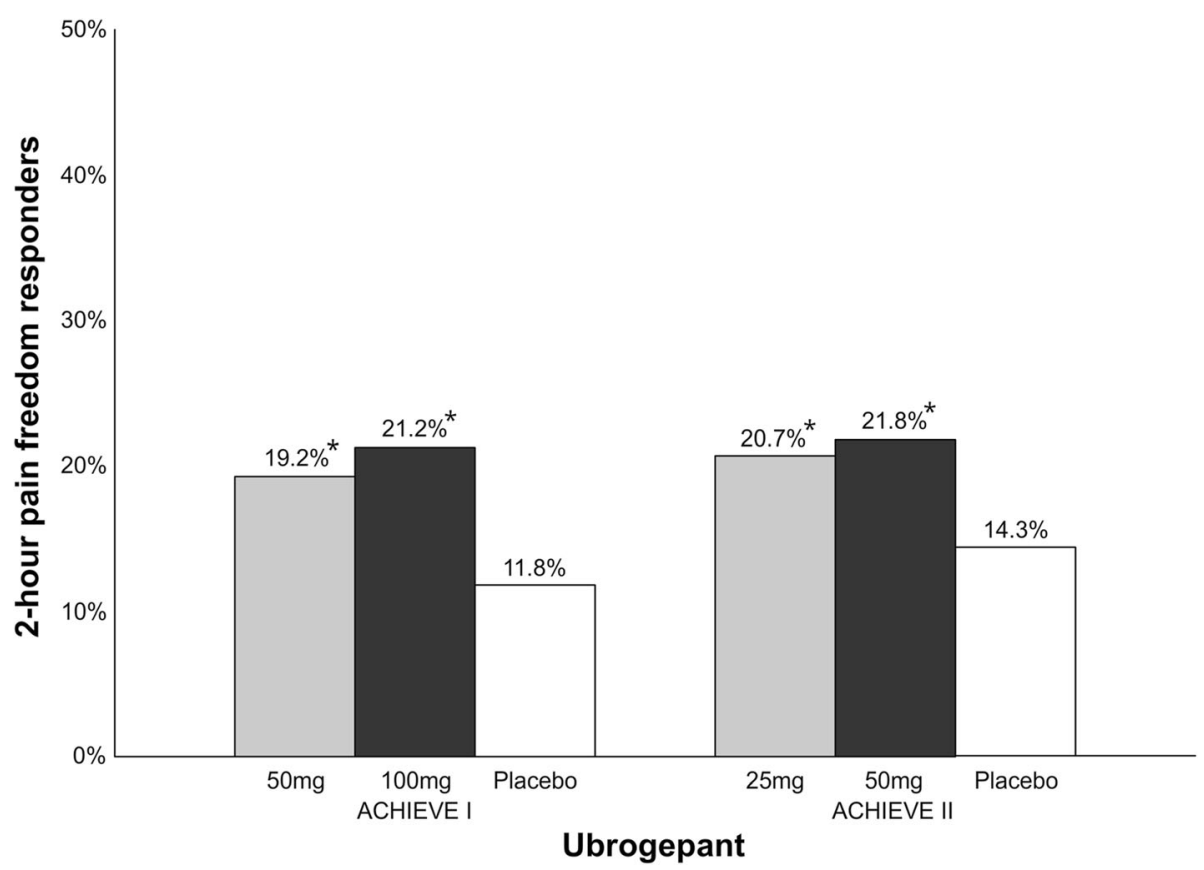

Fig. 5 Overview of patients (\%) achieving 2-h pain freedom in ubrogepant phase III clinical trials. *ACHIEVE I; 50 mg vs. placebo, $p=0.0023$; $100 \mathrm{mg}$ vs. placebo, $p=0.0003$. ACHIEVE II; $25 \mathrm{mg}$ vs. placebo, $p=0.0285 ; 50 \mathrm{mg}$ vs. placebo, $p=0.0129$ 


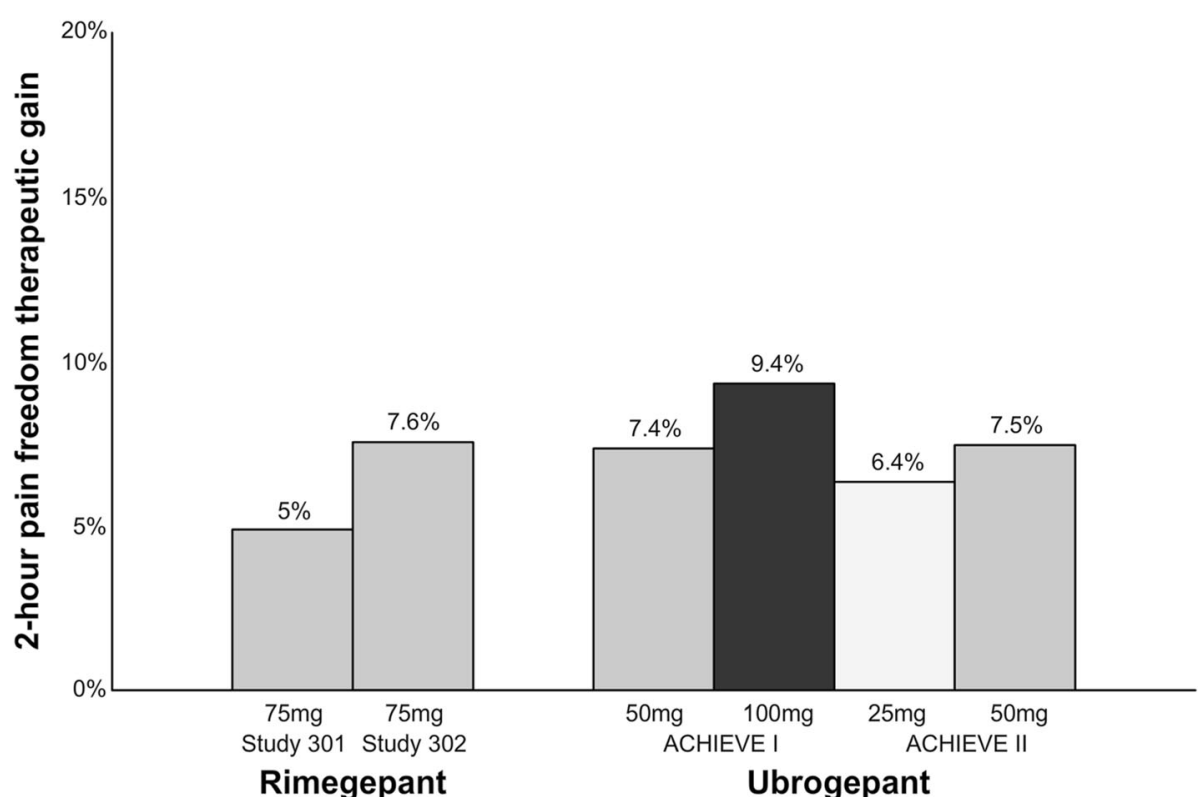

Fig. 6 Overview of the therapeutic gain* in 2-h pain freedom with gepants. A darker bar indicates a higher dose. *Therapeutic gain is defined as the difference between percentage of responders in active group compared to percentage of responders in placebo group

risk (or documented cardiovascular disease) or as second-line treatment if patients failed with triptans. The first gepants are expected to be approved by the FDA in 2019/2020 [44, 45].

\section{Novel prophylactic treatment targets Gepants}

Rimegepant (NCT03732638; phase II/III) and atogepant (NCT02848326, NCT03700320; phase II/III, phase III) are currently undergoing clinical trials in humans for prophylactic treatment of migraine but only data on atogepant has been released [46].

Preliminary data from the phase II clinical trial on atogepant have been reported in press releases [46]. The trial included 834 patients and was designed as a placebo-controlled dose ranging study with doses ranging from atogepant $10 \mathrm{mg}$ once a day to $60 \mathrm{mg}$ twice a day. All doses showed a significant reduction in mean monthly migraine days compared to placebo. The trial raised no concerns regarding hepatic or cardiovascular safety. Efficacy and safety data on atogepant need to be confirmed in phase III clinical trials.

\section{Anti-CGRP mAbs}

To date, four mAbs targeting the CGRP pathway have been developed (Table 3) and three of them have already been approved for the preventive treatment of migraine [47-49]. In the following we review data derived from recent phase III trials.

Erenumab is a humanized $\mathrm{IgG}_{2} \mathrm{mAb}$ that targets the CGRP receptor $[50,51]$ and administered as monthly subcutaneous injections of either $70 \mathrm{mg}$ or $140 \mathrm{mg}$. The mean $t_{\max }$ is 5.5 days and the plasma half-time is approximately $21-23$ days [52]. The $\mathrm{T}_{\max }$ corresponds with an early onset of effect with separation from placebo within the first week of treatment [53]. It has recently been approved for therapeutic use for the preventive treatment of migraine [47]. Data from two phase III clinical trials (ARISE and STRIVE) are presented in the following (Fig. 7). In ARISE, 577 patients were randomized

Table 3 Overview of anti-calcitonin-gene related (CGRP) (receptor) peptide monoclonal antibodies in order by target and alphabetical

\begin{tabular}{lllll}
\hline Drug & Target & Administration & Interval between administrations & Status \\
\hline Erenumab (AMG-334) & Receptor & Subcutaneous injection & 4 weeks & FDA approved; phase III clinical trials \\
Eptinezumab (ALD403) & Ligand & Intravenous infusion & 12 weeks & Phase III clinical trials \\
Fremanezumab (TEV-48125) & Ligand & Subcutaneous injection & 4 or 12 weeks & FDA approved; phase III clinical trials \\
Galcanezumab (LY2951742) & Ligand & Subcutaneous injection & 4 weeks & FDA approved; phase III clinical trials
\end{tabular}

*FDA: The US Food and Drug Administration 


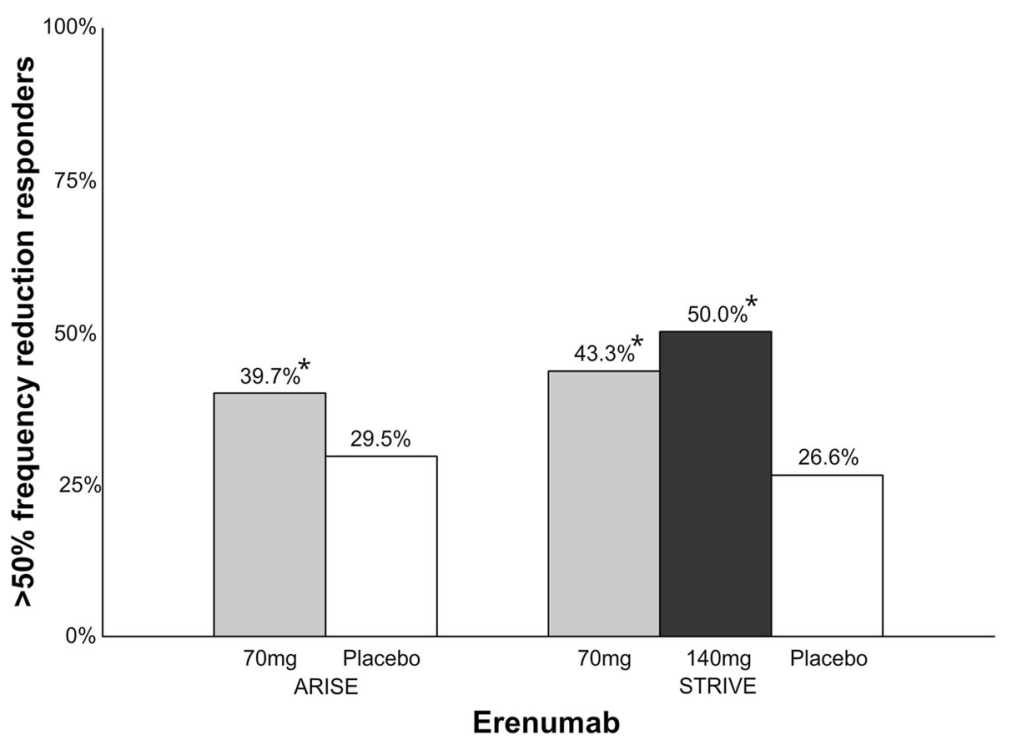

Fig. 7 Overview of patients (\%) achieving $>50 \%$ reduction in migraine days in phase III clinical trials with erenumab. A darker bar indicates a higher dose. *ARISE; $70 \mathrm{mg}$ vs. placebo, $p=0.010$. STRIVE; $70 \mathrm{mg}$ vs. placebo, $p<0.001 ; 140 \mathrm{mg}$ vs. placebo, $p<0.001$

to monthly injections of $70 \mathrm{mg}$ erenumab or placebo [54]. The percentage of patients achieving a $>50 \%$ reduction in monthly migraine days was $39.7 \%(p=0.010)$ in the active group and $29.5 \%$ in the placebo group. Adverse event rates were similar between erenumab and placebo. In STRIVE, 955 patients were randomized to monthly injections of erenumab $70 \mathrm{mg}, 140 \mathrm{mg}$ or placebo [55]. The percentage of patients achieving $>50 \%$ reduction in monthly migraine days was $43.3 \%$ (vs. placebo, $p<0.001$ ) with $70 \mathrm{mg}, 50.0 \%$ (vs. placebo, $\mathrm{p}<$ 0.001 ) with $140 \mathrm{mg}$ and $26.6 \%$ with placebo. There was no difference in adverse events between erenumab and placebo. The trial reported $8.0 \%$ of the $70 \mathrm{mg}$ group and $3.2 \%$ of the $140 \mathrm{mg}$ group creating anti-erenumab binding antibodies, however, only $5.6 \%$ of the patients were available for analysis.

Eptinezumab is a humanized $\operatorname{IgG}_{1} \mathrm{mAb}$ that binds to both $\alpha$ and $\beta$ forms of the human CGRP ligand [56]. The drug is administrated with intravenous infusions every 12 weeks. The plasma half-time of the drug is 31 days [56]. There is one completed phase III clinical trial (PROMISE I) [57], one ongoing phase III clinical trial (PROMISE II, NCT02974153) [58] and one ongoing safety open-label study (PREVAIL, NCT02985398) $[59,60]$. Preliminary data from the PROMISE I [61, 62] and PROMISE II [63] trials (Fig. 8) have been reported in press releases but not yet published in peer-reviewed journals. In PROMISE I, 888 patients were randomized to receive eptinezumab $30 \mathrm{mg}, 100 \mathrm{mg}, 300 \mathrm{mg}$ or placebo infusions once every 12 weeks $[61,62]$. For week $1-12$, percentage of patients achieving $>50 \%$ reduction in monthly migraine days was $49.8 \%$ (vs. placebo, $p=0.0085$ ) with $100 \mathrm{mg}, 56.3 \%$ (vs. placebo, $p=0.0001$ ) with $300 \mathrm{mg}$ and $37.4 \%$ with placebo [61]. For month 6-12, 70.7\% patients had a $>50 \%$ reduction in monthly migraine days compared to $58.7 \%$ for placebo [62]. Differences between doses were not reported for month 6-12. The most commonly reported adverse events across all eptinezumab groups were upper respiratory infection (10.5\%), nasopharyngitis (6.8\%), and sinusitis (3.6\%). In PROMISE II, 1072 patients were randomized to eptinezumab $100 \mathrm{mg}, 300 \mathrm{mg}$ or placebo [63]. The percentage of patients achieving $>50 \%$ reduction in monthly migraine days at week $1-12$ were $58 \%$ (vs. placebo, $p<0.0001$ ) with $100 \mathrm{mg}, 61 \%$ (vs. placebo, $\mathrm{p}<0.0001$ ) in 300 $\mathrm{mg}$ and 39\% with placebo. The incidence of adverse events was not statistically different from the placebo group.

Fremanezumab is a humanized $\operatorname{IgG}_{2}$ mAb that binds to both $\alpha$ and $\beta$ forms of the human CGRP ligand [64]. Fremanezumab has recently been approved for therapeutic use for the preventive treatment of migraine [48]. The drug is administered as subcutaneous injections with either monthly low-dose $225 \mathrm{mg}$ injections or quarterly high-dose $675 \mathrm{mg}$ injections. The $\mathrm{t}_{\max }$ is $5-7$ days and the plasma half-time of the drug is 31 days. The $t_{\max }$ corresponds with an early onset of effect with separation from placebo within the first week of treatment [65]. Results from two phase III clinical trials have been published in peer-reviewed journals (Fig. 9). In a phase III clinical trial [66], 1130 chronic migraine patients were randomized 1:1:1 to monthly low-dose $225 \mathrm{mg}$ injections, quarterly high-dose $675 \mathrm{mg}$ injections or placebo. Percentage of patients with $>50 \%$ reduction in monthly migraine days was $41 \%$ (vs. placebo, $p<0.001$ ) in monthly group, $38 \%$ (vs. placebo, $\mathrm{p}<0.001$ ) in quarterly group and $18 \%$ in the placebo group. Most common 


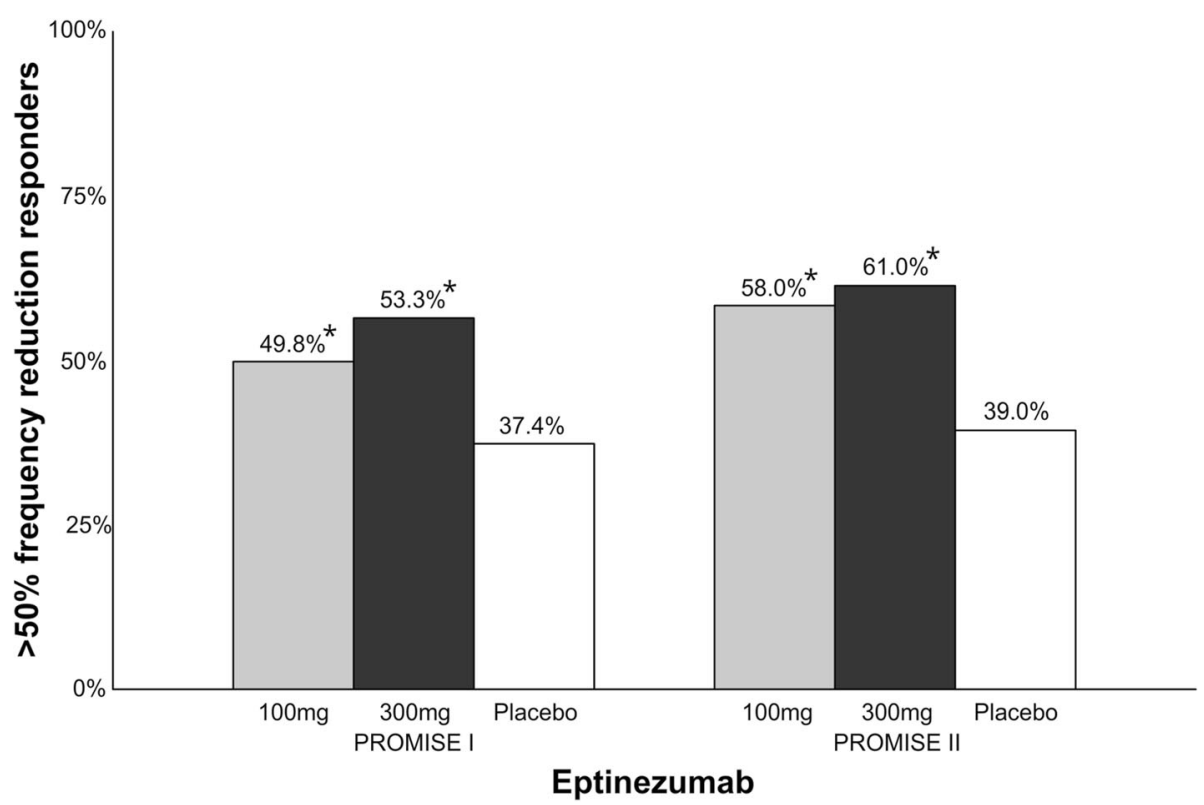

Fig. 8 Overview of patients (\%) achieving $>50 \%$ reduction in migraine days in phase III clinical trials with eptinezumab. A darker bar indicates a higher dose. *PROMISE I; $100 \mathrm{mg}$ vs. placebo, $p=0.0085 ; 300 \mathrm{mg}$ vs. placebo, $p=0.0001$. PROMISE II; $100 \mathrm{mg}$ vs. placebo, $p<0.0001 ; 300 \mathrm{mg}$ vs. placebo, $p<0.0001$

adverse event was injection site pain. Two (0.5\%) patients in the high-dose quarterly group developed anti-drug antibodies. Similar results were reported in another phase III clinical trial with 875 episodic migraine patients randomized to monthly low-dose $225 \mathrm{mg}$ injections or quarterly high-dose $675 \mathrm{mg}$ injections [67]. Percentage of patients with $>50 \%$ migraine frequency reduction was $47.7 \%$ (vs. placebo, $\mathrm{p}<0.001$ ) in monthly group, in $44.4 \%$ (vs. placebo, $\mathrm{p}<0.001$ ) quarterly group, and $27.9 \%$ in placebo group. Four patients $(1.4 \%)$ in the

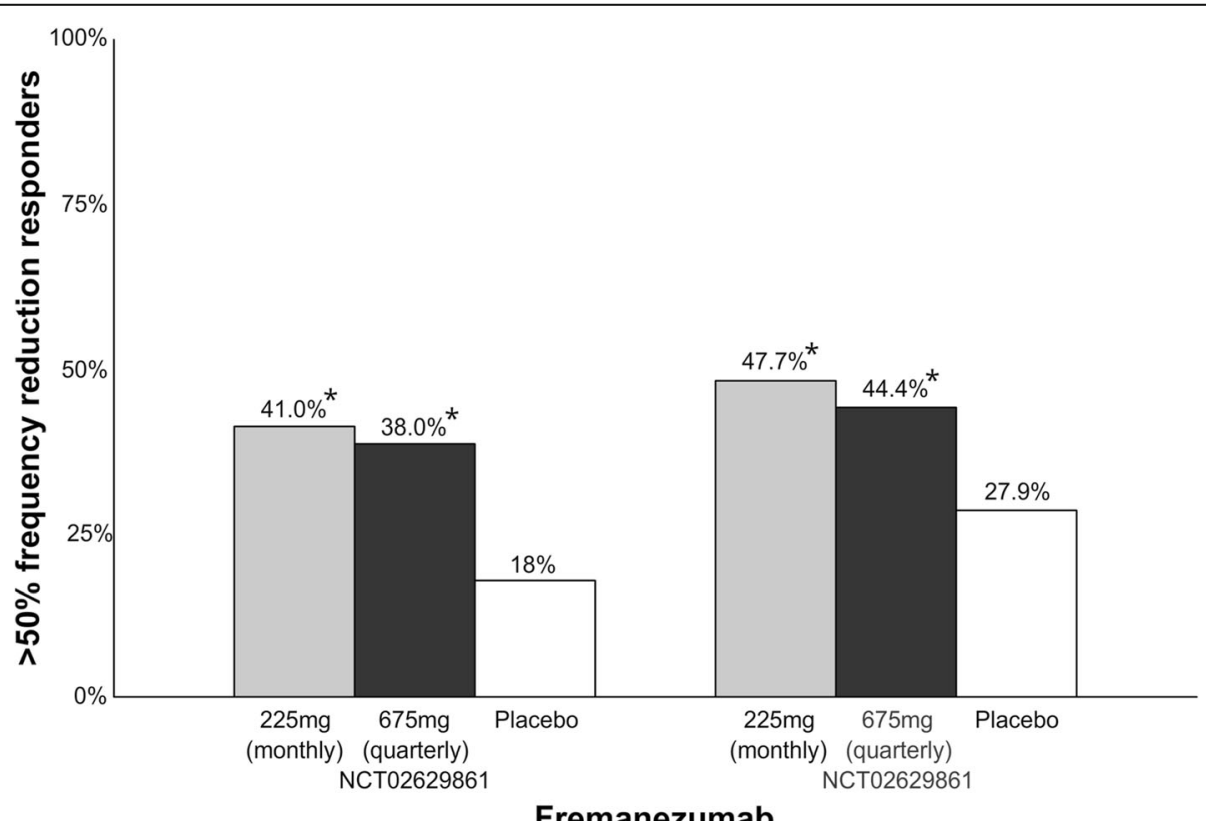

Fremanezumab

Fig. 9 Overview of patients (\%) achieving $>50 \%$ reduction in migraine days in phase III clinical trials with fremanezumab. A darker bar indicates a higher dose. *NCT02629861 (left); $225 \mathrm{mg}$ vs. placebo, $p<0.001 ; 675 \mathrm{mg}$ vs. placebo, $p<0.001$. NCT02629861 (right); $225 \mathrm{mg}$ vs. placebo, $p<0.001$; $675 \mathrm{mg}$ vs. placebo, $p<0.001$ 
low-dose monthly group developed anti-drug antibodies. The most common adverse events were also injection site reactions. In addition, post-hoc analyses show that fremanezumab is safe and effective as add-on treatment for migraine patients who is already on stable doses of other preventive migraine medication [68].

Galcanezumab is a humanized $\operatorname{IgG}_{4} \mathrm{mAb}$ that binds to both $\alpha$ and $\beta$ forms of the human CGRP ligand. Galcanezumab has recently been approved for the preventive treatment of migraine [49]. The drug is administered as monthly subcutaneous injections. The $t_{\max }$ is $7-13$ days and the plasma half-time of the drug is 28 days. Results from two phase III clinical trials (EVOLVE-1 and EVOLVE-2) have been published in peer-reviewed journals (Fig. 10). In EVOLVE-1, 1671 patients were randomized 1:1:2 to galcanezumab $120 \mathrm{mg}, 240 \mathrm{mg}$ or placebo [69]. Percentage of patients with $>50 \%$ migraine frequency reduction was $60.9 \%$ (vs. placebo, $p<0.001$ ) in $240 \mathrm{mg}$ group, $62.3 \%$ (vs. placebo, $\mathrm{p}<0.001$ ) in $120 \mathrm{mg}$ group and $38.6 \%$ in placebo group. The most common adverse event was injection site reactions. The number of treatment related adverse events was not statistically significant between the active and placebo groups. The percentage of patients who had anti-drug antibodies present after treatment were $5.2 \%$ in $240 \mathrm{mg}$ group, $3.5 \%$ in $120 \mathrm{mg}$ group and $1.7 \%$ in placebo group. In EVOLVE-2, 915 patients were randomized 1:1:2 to galcanezumab $120 \mathrm{mg}, 240 \mathrm{mg}$ or placebo [70]. Percentage of patients with $>50 \%$ migraine frequency reduction was
$59 \%$ (vs. placebo, $\mathrm{p}<0.001$ ) in $240 \mathrm{mg}$ group, $57 \%$ (vs. placebo, $\mathrm{p}<0.001)$ in $120 \mathrm{mg}$ group and $36 \%$ in placebo group. The most common adverse event was injection site reactions with a higher rate in the active groups compared to placebo. There was no difference in number of serious adverse events between the groups. The percentage of patients who had anti-drug antibodies present after treatment was $5.1 \%$ in $240 \mathrm{mg}$ group, $8.6 \%$ in $120 \mathrm{mg}$ group and $0.5 \%$ in placebo group. Treatment-emergent anti-drug antibodies had no impact on either safety or efficacy.

The introduction of anti-CGRP $\mathrm{mAbs}$ is a great advancement in migraine treatment because of responder rates with very favorable adverse event profiles. The highest percentage of patients with $>50 \%$ reduction in migraine days with each $\mathrm{mAb}$ ranges from $47.7 \%-62 \%$. This suggests a difference in the ratio of responders between the different mAbs. However, the therapeutic gain range is $22-23.7 \%$ indicating that anti-CGRP mAbs have a similar efficacy regardless of target (receptor or ligand) and administration form (subcutaneous or intravenous) (Fig. 11). The therapeutic gain with erenumab is increased with higher dosage (Fig. 11). Interestingly, the proportion of patients reaching $\geq 75 \%$ reduction from baseline at 3 months is also statistically significant with anti-CGRP mAbs compared to placebo. This suggests that there are subpopulations who greatly benefit from anti-CGRP mAbs. Efforts should be made to identify these patients and biomarkers that can predict treatment

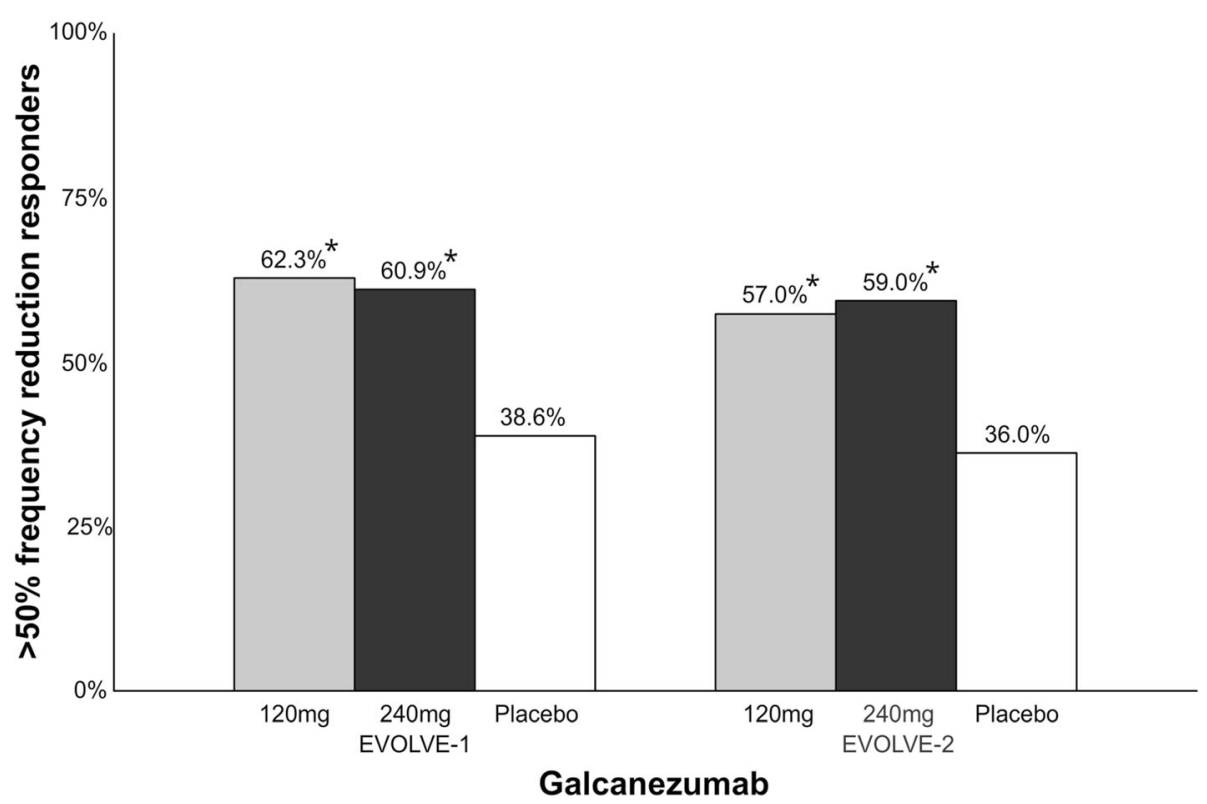

Fig. 10 Overview of patients (\%) achieving $>50 \%$ reduction in migraine days in phase III clinical trials with galcanezumab. A darker bar indicates a higher dose. ${ }^{*}$ EVOLVE-1; $120 \mathrm{mg}$ vs. placebo, $p<0.001 ; 240 \mathrm{mg}$ vs. placebo, $p<0.001$. EVOLVE-2; $120 \mathrm{mg}$ vs. placebo, $p<0.001 ; 240 \mathrm{mg}$ vs. placebo, $p<0.001$ 


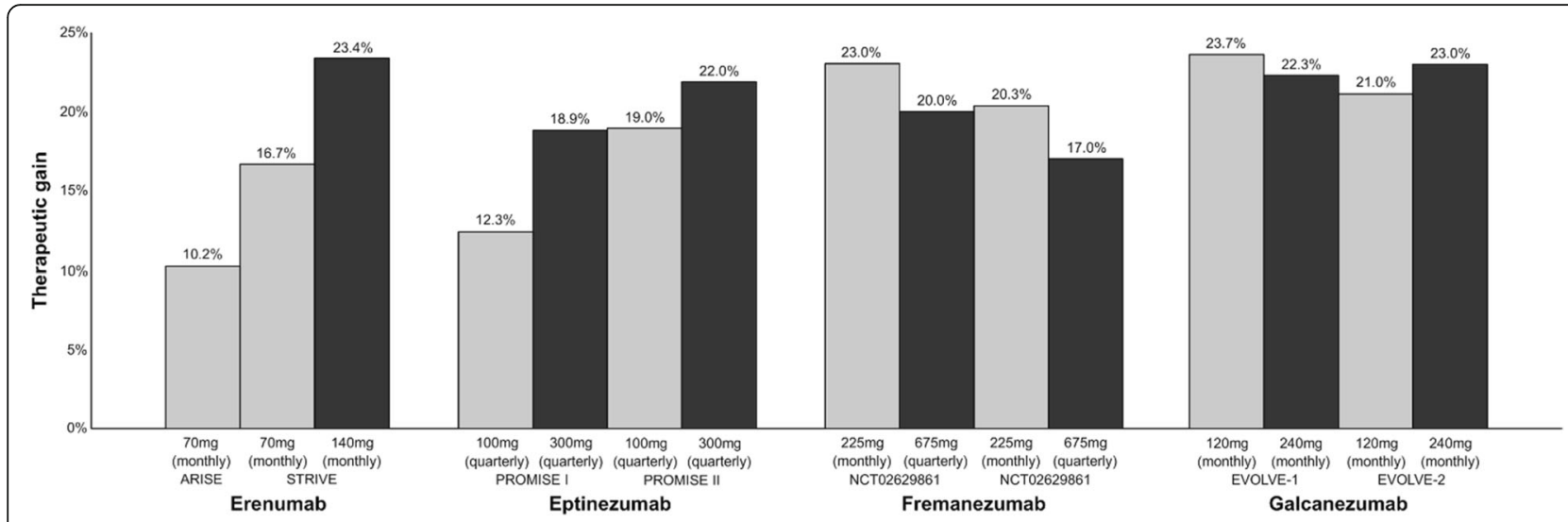

Fig. 11 Overview of the therapeutic gain* in percentage of patients with $>50 \%$ reduction in migraine days with anti-calcitonin gene-related peptide monoclonal antibodies. A darker bar indicates a higher dose. *Therapeutic gain is defined as the difference between percentage of patients in active group compared to percentage of patients in placebo group

response. The long-term effects (sustained efficacy and safety) of anti-CGRP mAbs remains to be investigated in real world studies. The rate of anti-drug binding antibodies is low in all trials reporting this parameter $[55,66,67,69,70]$. Adverse events related to the development of anti-drug antibodies has not been reported in anti-CGRP clinical trials. Whether anti-drug antibodies inactivate the clinical effect depends on the concentration of neutralizing anti-drug antibodies. Consequently, the detection of anti-drug antibodies itself is not a contraindication for treating with antiCGRP mAbs.

\section{Anti-pituitary adenylate cyclase-activating polypeptide mAbs} Pituitary adenylate cyclase-activating polypeptide (PACAP) belongs to the superfamily of glucagon/secretin peptides and two bioactive forms exist, PACAP38 and PACAP27 [71]. PACAP38 exists in the trigeminovascular system and deep brain structures amongst others [72-75]. PACAP38 mediates its effect through three receptors, pituitary adenylate cyclase-activating polypeptide type I $\left(\mathrm{PAC}_{1}\right), \mathrm{VPAC}_{1}$ and $\mathrm{VPAC}_{2}$. Like the CGRP pathway, these receptors cause an activation of adenylate cyclase leading to increased cAMP production [76]. Vasoactive intestinal peptide (VIP) has a similar structure to PACAP38 and affinity for $\mathrm{VPAC}_{1}$ and $\mathrm{VPAC}_{2}$ receptors. The two peptides differs in that
PACAP38 has a much higher affinity for the $\mathrm{PAC}_{1}$ receptor [77]. Furthermore, PACAP38 infusions can cause migraine-like attacks while VIP cannot $[78,79]$. Thus, only PACAP38 and the $\mathrm{PAC}_{1}$ receptor in this pathway are of interest as drug targets.

There are currently two mAbs, ALD1910 and AMG-301, in development for the PACAP38 pathway (Fig. 3) (Table 4). ALD1910 targets the PACAP38 ligand [80] and AMD-301 targets the $\mathrm{PAC}_{1}$ receptor [81]. ALD1910 is undergoing preclinical studies and AMG-301 has recently undergone a phase II clinical trial (NCT03238781). No results of either drug have been released so far but results from the AMG-301 trial is expected to be published mid-2019.

\section{Concluding remarks}

The development of ditans, gepants and anti-CGRP $\mathrm{mAbs}$ for the treatment of migraine is one of the greatest advances in the migraine field. Lasmiditan, rimegepant and ubrogepant will extend our therapeutic armamentarium for managing acute migraine attacks when triptans are not effective or contraindicated due to cardiovascular disorders. The mAbs against CGRP and its receptor have high responder rates with favorable adverse event profiles. Furthermore, the mAbs also offer convenient treatment regimens of 4- or 12-week intervals. These factors will contribute to a better adherence.

Table 4 Overview of anti-pituitary adenylate cyclase-activating polypeptide/pituitaryadenylate cyclase 1 (PACAP/PAC $)$ monoclonal antibodies

\begin{tabular}{lllll}
\hline Drug & Target & Administration & Interval between administrations & Status \\
\hline ALD1910 & Ligand & N/A & N/A & Preclinical phase \\
AMG-301 & Receptor & Subcutaneous injection & 4 weeks & Phase II clinical trials \\
\hline
\end{tabular}


Given that approximately $40-50 \%$ of migraine patients do not respond to mAbs, future studies should focus on identification of biomarkers that can predict treatment response. Collectively, novel migraine therapies represent a major progress in migraine treatment and will undoubtedly transform headache medicine.

\section{Abbreviations}

5-HT receptor: 5-hydroxytryptamine receptor; CGRP: Calcitonin gene-related peptide; $\mathrm{mAb}$ : Monoclonal antibody; $\mathrm{PAC}_{1}$ : Pituitary adenylate cyclaseactivating polypeptide type I; PACAP: Pituitary adenylate cyclase-activating polypeptide; RCT: Randomized controlled trial

\section{Acknowledgements}

The APCs (article processing charges) for the articles in this thematic series 'The Changing faces of migraine' were made possible through independent educational sponsorship by Eli Lilly. Eli Lilly provided the funds through an educational grant which included enduring materials within the context of a symposium at the 12th European Headache Federation Congress in September 2018, chaired by Paolo Martelletti. This grant was provided to Springer Healthcare IME who organized the symposium and all of the enduring materials. Three of the articles in this thematic series were developed from content presented at the symposium. Eli Lilly were not involved in the planning of the thematic series, the selection process for topics, nor in any peer review or decision-making processes.

The articles have undergone the journal's standard peer review process overseen by the Editor-in-Chief. For articles where the Editor-in-Chief is an author, the peer review process was overseen by one of the other Editors responsible for this thematic series.

\section{Funding}

Not applicable.

\section{Availability of data and materials}

Data sharing is not applicable to this article as no datasets were generated or analysed during the current study.

\section{Authors' contributions}

All authors contributed with data interpretation, drafting and revision of the manuscript for intellectual content. All authors read and approved the final manuscript.

\section{Ethics approval and consent to participate}

Not applicable.

\section{Consent for publication}

Not applicable.

\section{Competing interests}

MA is a consultant or scientific advisor for Allergan, Amgen, Alder, Eli Lilly, Novartis and Teva, principal investigator for: Amgen 20,120,178 (Phase 2), 20,120,295 (Phase 2), 20,130,255 (Open label extension), 20,120,297 (Phase 3), 20,150,308 (Phase 2), ElectroCore GM-11 gamma-Core-R, TEVA TV48125-CNS30068 (Phase 3), Novartis CAMG334A2301 (Phase 3) and Alder PROMISE-2. MA has no ownership interest and does not hold stock in any pharmaceutical company. MA serves as associated editor of Cephalalgia and co-editor of the Journal of Headache and Pain and is Editor for the thematic series 'The changing face of migraine'. TPD and SG report no competing interests.

\section{Publisher's Note}

Springer Nature remains neutral with regard to jurisdictional claims in published maps and institutional affiliations.

Received: 26 December 2018 Accepted: 18 February 2019 Published online: 17 April 2019

\section{References}

1. Ashina M, Hansen JM, BO ÁD et al (2017) Human models of migraine short-term pain for long-term gain. Nat Rev Neurol 13:713-724
2. Schytz HW, Schoonman GG, Ashina M (2010) What have we learnt from triggering migraine? Curr Opin Neurol 23:259-265

3. Goadsby P, Lipton R, Ferrari M (2002) Migraine- current understanding and treatment. N Engl J Med 346:257-270

4. Humphrey PP, Feniuk W, Perren MJ et al (1990) Serotonin and migraine. Ann N Y Acad Sci 600:587-598 discussion 598-600

5. Goadsby PJ (2009) The vascular theory of migraine--a great story wrecked by the facts. Brain. 132:6-7

6. Hou M, Kanje M, Longmore J et al (2001) 5-HT(1B) and 5-HT(1D) receptors in the human trigeminal ganglion: co-localization with calcitonin gene-related peptide, substance $\mathrm{P}$ and nitric oxide synthase. Brain Res 909:112-120

7. Gomez-Mancilla B, Cutler NR, Leibowitz MT et al (2001) Safety and efficacy of PNU-142633, a selective 5-HT1D agonist, in patients with acute migraine. Cephalalgia. 21:727-732

8. Mitsikostas DD, Tfelt-Hansen P (2012) Targeting to 5-HT1F receptor subtype for migraine treatment: lessons from the past, implications for the future. Cent Nerv Syst Agents Med Chem 12:241-249

9. Ma QP (2001) Co-localization of 5-HT(1B/1D/1F) receptors and glutamate in trigeminal ganglia in rats. Neuroreport. 12:1589-1591

10. Nilsson T, Longmore J, Shaw D et al (1999) Characterisation of 5-HT receptors in human coronary arteries by molecular and pharmacological techniques. Eur J Pharmacol 372:49-56

11. Maclntyre PD, Bhargava B, Hogg KJ et al (1993) Effect of subcutaneous sumatriptan, a selective $5 \mathrm{HT} 1$ agonist, on the systemic, pulmonary, and coronary circulation. Circulation. 87:401-405

12. MaassenVanDenBrink A, Reekers M, Bax WA et al (1998) Coronary sideeffect potential of current and prospective antimigraine drugs. Circulation. 98:25-30

13. O'Connor P, Gladstone P (1995) Oral sumatriptan-associated transmural myocardial infarction. Neurology. 45:2274-2276

14. Jayamaha JEL, Street MK (1995) Fatal cerebellar infarction in a migraine sufferer whilst receiving sumatriptan. Intensive Care Med 21:82-83

15. Abbrescia VD, Pearlstein L, Kotler M (1997) Sumatriptan-associated myocardial infarction: report of case with attention to potential risk factors. J Am Osteopath Assoc 97:162-164

16. Vila-Pueyo M (2018) Targeted 5-HT1F therapies for migraine. Neurotherapeutics. 15:291-303

17. Goldstein DJ, Roon Kl, Offen WW et al (2001) Selective seratonin 1F (5HT(1F)) receptor agonist LY334370 for acute migraine: a randomised controlled trial. Lancet 358:1230-1234

18. Rizzoli PB (2014) Emerging therapeutic options for acute migraine: focus on the potential of lasmiditan. Neuropsychiatr Dis Treat 10:547-552

19. Nelson DL, Phebus LA, Johnson KW et al (2010) Preclinical pharmacological profile of the selective 5-HT1F receptor agonist lasmiditan. Cephalalgia. 30: 1159-1169

20. Oswald JC, Schuster NM (2018) Lasmiditan for the treatment of acute migraine: a review and potential role in clinical practice. J Pain Res 11: 2221-2227

21. Lasmiditan Compared to Placebo in the Acute Treatment of Migraine: (SAMURAl) https://clinicaltrials.gov/ct2/show/NCT02439320 (accessed 31 Oct 2018)

22. Three Doses of Lasmiditan (50 mg, $100 \mathrm{mg}$ and $200 \mathrm{mg}$ ) Compared to Placebo in the Acute Treatment of Migraine (SPARTAN). DOI: https:// clinicaltrials.gov/ct2/show/NCT02605174

23. An Open-label, Long-term, Safety Study of Lasmiditan for the Acute Treatment of Migraine (GLADIATOR)https://clinicaltrials.gov/ct2/show/ NCT02565186 (accessed 31 Oct 2018)

24. Kuca B, Silberstein SD, Wietecha L et al (2018) Lasmiditan is an effective acute treatment for migraine: a phase 3 randomized study. Neurology. 91: e2222-e2232

25. Lilly Announces Positive Results for Second Phase 3 Study of Lasmiditan for the Acute Treatment of Migraine https://investor.lilly.com/static-files/ 15cf1 efc-da8f-485c-9001-6ff3b432b129

26. CoLucid Pharmaceuticals Provides Interim Update on GLADIATOR https:// globenewswire.com/news-release/2016/09/19/872772/0/en/CoLucidPharmaceuticals-Provides-Interim-Update-on-GLADIATOR.html (accessed 1 Nov 2018)

27. CoLucid Pharmaceuticals Announces Achievement of Both Primary and Key Secondary Endpoints in the SAMURAI Phase 3 Pivotal Trial of Lasmiditan in Migraine https://globenewswire.com/news-release/2016/09/06/869611/0/ en/CoLucid-Pharmaceuticals-Announces-Achievement-of-Both-Primary-and- 
Key-Secondary-Endpoints-in-the-SAMURAI-Phase-3-Pivotal-Trial-ofLasmiditan-in-Migraine.html (accessed 31 Oct 2018)

28. Derry CJ, Derry S, Moore RA (2014) Sumatriptan (all routes of administration) for acute migraine attacks in adults - overview of Cochrane reviews. Cochrane database Syst Rev:CD009108

29. Olesen J, Diener H-C, Husstedt IW et al (2004) Calcitonin gene-related peptide receptor antagonist BIBN 4096 BS for the acute treatment of migraine. N Engl J Med 350:1104-1110

30. Ho TW, Connor KM, Zhang Y et al (2015) Randomized controlled trial of the CGRP receptor antagonist telcagepant for prevention of headache in women with perimenstrual migraine. Cephalalgia. 36:148-161

31. Goadsby PJ, Holland PR, Martins-Oliveira M et al (2017) Pathophysiology of migraine: a disorder of sensory processing. Physiol Rev 97:553-622

32. Luo G, Chen L, Conway CM et al (2012) Discovery of (5S,6S,9R)-5-amino-6(2,3-difluorophenyl)-6,7,8,9-tetrahydro-5H-cyclohepta[b]pyridin-9-yl 4-(2-oxo2,3-dihydro-1 H-imidazo [4,5-b]pyridin-1-yl)piperidine-1-carboxylate (BMS927711): an oral calcitonin gene-related peptide (CGRP) antagonist in c. J Med Chem 55:10644-10651

33. Croop R, Ivans A, Stock D, et al. A Phase 1 Study To Evaluate The Bioequivalence Of Oral Tablet And Orally Dissolving Tablet Formulations Of Rimegepant, A Small Molecule Cgrp Receptor Antagonist. In: 17th Biennial Migraine Trust International Symposium, p. 116

34. Biohaven Announces Successful Achievement of Both Co-Primary Regulatory Endpoints in Two Pivotal Phase 3 Trials of Rimegepant an Oral CGRP Receptor Antagonist for the Acute Treatment of Migraine https:// biohavenpharma.com/wp-content/uploads/2018/03/CONFIDENTIALBIOHAVEN-PRESS-RELEASE-FINAL-V2.pdf (accessed 16 Oct 2018)

35. Biohaven Announces Robust Clinical Data with Single Dose Rimegepant That Defines Acute and Durable Benefits to Patients: The First Oral CGRP Receptor Antagonist to Deliver Positive Data on Pain Freedom and Most Bothersome Symptomin Two Pivotal Phase 3 Tri https://biohavenpharma. com/wp-content/uploads/2018/04/NON-CONFIDENTIAL-PRESS-RELEASERimegepant-Secondary-Endpoints-Show-Comprehensive-and-DurableClinical-Benefits-FINAL.pdf (accessed 16 Oct 2018)

36. Voss T, Lipton RB, Dodick DW et al (2016) A phase llb randomized, doubleblind, placebo-controlled trial of ubrogepant for the acute treatment of migraine. Cephalalgia. 36:887-898

37. Allergan Announces Positive Top Line Phase 3 Results for Ubrogepant - an Oral CGRP Receptor Antagonist for the Acute Treatment of Migraine https:// www.allergan.com/news/news/thomson-reuters/allergan-announcespositive-top-line-phase-3-resul (accessed 17 Oct 2018)

38. Allergan Announces Second Positive Phase 3 Clinical Trial for Ubrogepant -an Oral CGRP Receptor Antagonist for the Acute Treatment of Migraine https:/www.allergan.com/News/News/Thomson-Reuters/AllerganAnnounces-Second-Positive-Phase-3-Clinica (accessed 17 Oct 2018)

39. Tfelt-Hansen P, Do T (2012) Is oral telcagepant a relatively slowly acting drug? A mini-review of 4 RCTs. Cephalalgia. 32:1-30

40. Tfelt-Hansen P, Loder E. The Emperor's New Gepants: Are the Effects of the New Oral CGRP Antagonists Clinically Meaningful? Headache. Epub ahead of print 19 November 2018. DOI: https://doi.org/10.1111/head.13444

41. Brain SD, Grant AD (2004) Vascular actions of calcitonin gene-related peptide and adrenomedullin. Physiol Rev 84:903-934

42. Petersen KA, Birk S, Lassen LH et al (2005) The CGRP-antagonist, BIBN4096BS does not affect cerebral or systemic haemodynamics in healthy volunteers. Cephalalgia. 25:139-147

43. Petersen KA, Lassen LH, Birk S et al (2005) BIBN4096BS antagonizes human alpha-calcitonin gene related peptide-induced headache and extracerebral artery dilatation. Clin Pharmacol Ther 77:202-213

44. Rimegepant For Acute and Preventive Treatment of Migraine https:// www.biohavenpharma.com/science-pipeline/cgrp/rimegepant (accessed 12 Feb 2019)

45. Allergan Announces Completion of Two Positive Safety Studies for Ubrogepant - an Oral CGRP Receptor Antagonist for the Acute Treatment of Migraine https://www.allergan.com/news/news/thomson-reuters/allerganannounces-completion-of-two-positive-safe (accessed 12 Feb 2019)

46. Allergan's Oral CGRP Receptor Antagonist Atogepant Demonstrates Robust Efficacy and Safety in Episodic Migraine Prevention in a Phase 2b/3 Clinical Trial https://www.allergan.com/news/news/thomson-reuters/allergan-s-oralcgrp-receptor-antagonist-atogepant (accessed 20 Nov 2018)

47. Novartis and Amgen announce FDA approval of Aimovig(TM) (erenumab), a novel treatment developed specifically for migraine preventionhttps://www. novartis.com/news/media-releases/novartis-and-amgen-announce-fdaapproval-aimovigtm-erenumab-novel-treatment-developed-specificallymigraine-prevention (accessed 20 September 2018)

48. Teva Announces U.S. Approval of AJOVY'M (fremanezumab-vfrm) Injection, the First and Only Anti-CGRP Treatment with Both Quarterly and Monthly Dosing for the Preventive Treatment of Migraine in Adultshttps://www.tevapharm.com/news/teva_announces_u_s_approval_ of_ajovytm_fremanezumab_vfrm_injection_the_first_and_only_anti_ cgrp_treatment_with_both_quarterly_and_monthly_dosing_for_the_ preventive_treatment_of_migraine_in_adults_09_18.aspx (accessed 20 September 2018)

49. Lilly's Emgality ${ }^{\top M}$ (galcanezumab-gnlm) Receives U.S. FDA Approval for the Preventive Treatment of Migraine in Adultshttps://investor.lilly.com/newsreleases/news-release-details/lillys-emgalitytm-galcanezumab-gn/m-receivesus-fda-approval (accessed 31 October 2018)

50. Giamberardino MA, Affaitati G, Costantini R et al (2017) Calcitonin generelated peptide receptor as a novel target for the management of people with episodic migraine: current evidence and safety profile of erenumab. J Pain Res 10:2751-2760

51. Shi L, Lehto SG, Zhu DXD et al (2016) Pharmacologic characterization of AMG 334, a potent and selective human monoclonal antibody against the calcitonin gene-related peptide receptor. J Pharmacol Exp Ther 356:223-231

52. de Hoon J, Van Hecken A, Vandermeulen C et al (2018) Phase I, randomized, double-blind, placebo-controlled, single-dose, and multipledose studies of Erenumab in healthy subjects and patients with migraine. Clin Pharmacol Ther 103:815-825

53. Schwedt T, Reuter U, Tepper S et al (2018) Early onset of efficacy with erenumab in patients with episodic and chronic migraine. J Headache Pain 19:92

54. Dodick DW, Ashina M, Brandes JL et al (2018) ARISE: a phase 3 randomized trial of erenumab for episodic migraine. Cephalalgia. 38:1026-1037

55. Goadsby PJ, Reuter U, Hallström Y et al (2017) A controlled trial of Erenumab for episodic migraine. N Engl J Med 377:2123-2132

56. Dodick DW, Goadsby PJ, Silberstein SD et al (2014) Safety and efficacy of ALD403, an antibody to calcitonin gene-related peptide, for the prevention of frequent episodic migraine: a randomised, double-blind, placebo-controlled, exploratory phase 2 trial. Lancet Neurol 13:1100-1107

57. A Multicenter Assessment of ALD403 in Frequent Episodic Migraine (PROMISE 1) https://clinicaltrials.gov/ct2/show/NCT02559895 (accessed 31 October 2018)

58. Evaluation of ALD403 (Eptinezumab) in the Prevention of Chronic Migraine (PROMISE 2) https://clinicaltrials.gov/ct2/show/NCT02974153

59. Eptinezumab Alder Biopharmaceuticals https://www.alderbio.com/pipeline/ eptinezumab/ (accessed 19 Oct 2018)

60. An Open Label Trial of ALD403 (Eptinezumab) in Chronic Migraine https:// clinicaltrials.gov/ct2/show/NCT02985398 (accessed 31 Oct 2018)

61. Alder BioPharmaceuticals Announces Positive Eptinezumab Phase 3 Results for Prevention of Frequent Episodic Migraine https://investor.alderbio.com/ news-releases/news-release-details/alder-biopharmaceuticals-announcespositive-eptinezumab-phase-3 (accessed 19 Oct 2018)

62. Alder BioPharmaceuticals ${ }^{\circledR}$ Presents New 12-Month Data of Eptinezumab in PROMISE 1 Phase 3 Trial Showing Long-Term Reduction in Episodic Migraine https://investor.alderbio.com/news-releases/news-release-details/ alder-biopharmaceuticalsr-presents-new-12-month-data-eptinezumab (accessed 31 Oct 2018)

63. PROMISE 2 Top-Line Data Results https://investor.alderbio.com/static-files/ b943669f-4b0c-4e10-a177-36e7974e9057 (accessed 30 Oct 2018)

64. Bigal ME, Escandon R, Bronson M et al (2014) Safety and tolerability of LBR101, a humanized monoclonal antibody that blocks the binding of CGRP to its receptor: results of the phase 1 program. Cephalalgia. 34:483-492

65. Bigal ME, Dodick DW, Krymchantowski AV et al (2016) TEV-48125 for the preventive treatment of chronic migraine: efficacy at early time points. Neurology. 87:41-48

66. Silberstein SD, Dodick DW, Bigal ME et al (2017) Fremanezumab for the preventive treatment of chronic migraine. N Engl J Med 377:2113-2122

67. Dodick DW, Silberstein SD, Bigal ME et al (2018) Effect of Fremanezumab compared with placebo for prevention of episodic migraine: a randomized clinical trial. JAMA. 319:1999-2008

68. Cohen JM, Dodick DW, Yang R et al (2017) Fremanezumab as add-on treatment for patients treated with other migraine preventive medicines. Headache. 57:1375-1384 
69. Stauffer VL, Dodick DW, Zhang Q et al (2018) Evaluation of Galcanezumab for the prevention of episodic migraine: the EVOLVE-1 randomized clinical trial. JAMA Neurol 75:1080-1088

70. Skljarevski V, Matharu M, Millen BA et al (2018) Efficacy and safety of galcanezumab for the prevention of episodic migraine: results of the EVOLVE-2 phase 3 randomized controlled clinical trial. Cephalalgia. 38:1442-1454

71. Miyata A, Jiang L, Dahl RD et al (1990) Isolation of a neuropeptide corresponding to the $\mathrm{N}$-terminal 27 residues of the pituitary adenylate cyclase activating polypeptide with 38 residues (PACAP38). Biochem Biophys Res Commun 170:643-648

72. Mulder H, Uddman R, Moller K et al (1994) Pituitary adenylate cyclase activating polypeptide expression in sensory neurons. Neuroscience. 63:307-312

73. Uddman R, Tajti J, Hou M et al (2002) Neuropeptide expression in the human trigeminal nucleus caudalis and in the cervical spinal cord C1 and C2. Cephalalgia. 22:112-116

74. Dun EC, Huang RL, Dun SL et al (1996) Pituitary adenylate cyclase activating polypeptide-immunoreactivity in human spinal cord and dorsal root ganglia. Brain Res 721:233-237

75. Ghatei MA, Takahashi K, Suzuki Y et al (1993) Distribution, molecular characterization of pituitary adenylate cyclase-activating polypeptide and its precursor encoding messenger RNA in human and rat tissues. J Endocrinol 136:159-166

76. Vaudry D, Gonzalez BJ, Basille M et al (2000) Pituitary adenylate cyclaseactivating polypeptide and its receptors: from structure to functions. Pharmacol Rev 52:269-324

77. Harmar AJ, Fahrenkrug J, Gozes I et al (2012) Pharmacology and functions of receptors for vasoactive intestinal peptide and pituitary adenylate cyclase-activating polypeptide: IUPHAR review 1. Br J Pharmacol 166:4-17

78. Schytz HW, Birk S, Wienecke T et al (2009) PACAP38 induces migraine-like attacks in patients with migraine without aura. Brain. 132:16-25

79. Rahmann A, Wienecke T, Hansen JM et al (2008) Vasoactive intestinal peptide causes marked cephalic vasodilation, but does not induce migraine. Cephalalgia. 28:226-236

80. ALD1910: Advancing the Science for Migraine Prevention https://www. alderbio.com/pipeline/ald1910/ (accessed 31 Oct 2018)

81. Study to Evaluate the Efficacy and Safety of AMG 301 in Migraine Prevention https://clinicaltrials.gov/ct2/show/NCT03238781 (accessed 31 Oct 2018)

Ready to submit your research? Choose BMC and benefit from:

- fast, convenient online submission

- thorough peer review by experienced researchers in your field

- rapid publication on acceptance

- support for research data, including large and complex data types

- gold Open Access which fosters wider collaboration and increased citations

- maximum visibility for your research: over $100 \mathrm{M}$ website views per year

At $\mathrm{BMC}$, research is always in progress.

Learn more biomedcentral.com/submissions 